\title{
Increase in Equilibrium Price by Fast Oscillations
}

\author{
Babar Ahmad ${ }^{1}$ and Khalid Iqbal Mahr ${ }^{2}$ \\ ${ }^{1}$ COMSATS Institute of Information Technology, Islamabad 44000, Pakistan \\ ${ }^{2}$ Muhammad Ali Jinnah University, Islamabad 44000, Pakistan \\ Correspondence should be addressed to Babar Ahmad; babar.sms@gmail.com \\ Received 15 January 2014; Revised 25 April 2014; Accepted 25 April 2014; Published 20 May 2014 \\ Academic Editor: Ivo Petras
}

Copyright (C) 2014 B. Ahmad and K. I. Mahr. This is an open access article distributed under the Creative Commons Attribution License, which permits unrestricted use, distribution, and reproduction in any medium, provided the original work is properly cited.

The dynamics of a market can be described by a differential equation. Using the concept of fast oscillation, the system (typical market) can also oscillate around a new equilibrium price, with an increase. Previously that increase was established by applying harmonic force. In present work, harmonic force is replaced by an arbitrary periodic force with zero mean. Hence the increase in equilibrium price can be controlled by varying the external arbitrary periodic force.

\section{Introduction}

The statistical physics and nonlinear dynamics can be employed as tools in economics and social studies [1] to build up econophysics [2] and statistical finance [3]. Examples are trading and price formation [4], excess and clustering of stochastic volatility $[5,6]$, investigation of scaling [7] of the competitive equilibrium $[8,9]$, and role of noise to increase stability $[10,11]$ in many physical systems.

Using Kapitza method [12], Landau and Lifshitz discussed the stability of the inverted pendulum under fast oscillation. He showed that when the suspension of a pendulum has vertical modulation with harmonic force, the position $\phi=0$ is always stable and $\phi=\pi$ is conditionally stable [13].

Using this approach in the market, Holyst and Wojciechowski have shown that due to fast oscillation a new equilibrium price can occur. Using external harmonic force, this new equilibrium price is proportional to the difference $D^{\prime \prime}(p)-S^{\prime \prime}(p)$. Hence due to fast oscillation, the equilibrium price of the market will increase [14].

In 2009 Ahmad and Borisenok extended the idea of stability for arbitrary periodic force and stabilized the inverted pendulum with relatively low frequency. They used periodic kicking pulses in place of harmonic force. Then the conditional stable point is controlled by varying external periodic force [15].
In this paper, the dynamics of the market is studied along with external arbitrary periodic force, with zero mean. Then another equilibrium price can be established with an increase. This increase can be controlled by applying a particular periodic force.

\section{Kapitza Method for Arbitrary Periodic Force}

A particle of mass $m$ is moving under a force due to timeindependent potential $U(x)$ :

$$
f_{1}(x)=-\frac{d U}{d x}
$$

and a periodic fast oscillating force with zero mean. This fast oscillating force in Fourier expansion is

$$
\begin{aligned}
f_{2} & (x, t) \\
& =\sum_{k=1}^{\infty}\left[a_{k}(x) \cos (k \omega t)+b_{k}(x) \sin (k \omega t)\right] .
\end{aligned}
$$

Here $\omega \equiv 2 \pi / T \gg 2 \pi / T_{0} \equiv \omega_{0}$ and $\omega_{0}$ is the frequency of motion due to $f_{1}$. The mean value of a function is denoted by bar and is defined as

$$
\bar{f}=\frac{1}{T} \int_{0}^{T} f(x, t) d t
$$




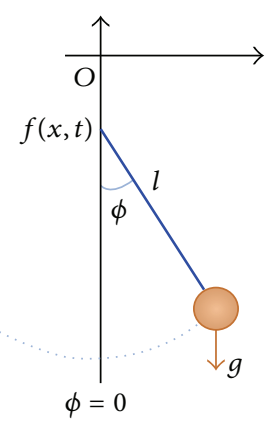

FIgURE 1: Vertical modulation.

Also the Fourier coefficient $a_{0}$ is

$$
a_{0}(x)=\frac{2}{T} \int_{0}^{T} f_{2}(x, t) d t .
$$

Since we are choosing a force with zero mean, then from (3) and (4) it follows that

$$
\bar{f} \cong a_{0}=0 .
$$

In (2), $a_{k}$ and $b_{k}$ are the Fourier coefficients, given by

$$
\begin{aligned}
& a_{k}(x)=\frac{2}{T} \int_{0}^{T} f_{2}(x, t) \cos k \omega t d t \\
& b_{k}(x)=\frac{2}{T} \int_{0}^{T} f_{2}(x, t) \sin k \omega t d t .
\end{aligned}
$$

Due to (1) and (2) the equation of motion is

$$
m \ddot{x}=f_{1}(x)+f_{2}(x, t) .
$$

Equation (7) represents that the system has two motions at a time, one along a smooth path and the other small but fast oscillations. So the path can be written as

$$
x(t)=X(t)+\xi(t) .
$$

Here $X(t)$ represents smooth path and $\xi(t)$ represents fast oscillations. By averaging procedure the effective potential energy function is [15]

$$
U_{\mathrm{eff}}=U+\frac{1}{4 m \omega^{2}} \sum_{k=1}^{\infty} \frac{\left(a_{k}^{2}+b_{k}^{2}\right)}{k^{2}} .
$$

2.1. Stability. Kapitza pendulum modulated vertically by arbitrary periodic force $f(x, t)$ is illustrated in Figure 1 . Where $g$ is acceleration due to gravity, $l$ is the length of the massless string. The system is stabilized by minimizing (9). The position $\phi=0$ is always stable (see Figure 2(a)), while the position $\phi=\pi$ is stable if $\omega^{2}>0.5 g l \sum_{k=1}^{\infty}\left(k^{2} /\left(a_{k}^{2}+b_{k}^{2}\right)\right)$ (see Figure 2(b)). The sum $\sum_{k=1}^{\infty}\left(\left(a_{k}^{2}+b_{k}^{2}\right) / k^{2}\right)$ is associated with external force. It follows that due to fast oscillation the inverted position may also be stable [15].

The concept of fast oscillation is used in the market. Then another equilibrium price, with an increase, can be selected. This increase can be controlled by varying the external periodic force.

\section{Effect of Fast Oscillation on Equilibrium Price}

Almost every commodity has more price than its actual price, as some external factors are involved in that increase. In this paper, a mathematical approach is presented: how an authority can increase/decrease the price of the commodity.

Let $p(t)$ be the commodity price at any time $t$. The time rate of change of $p(t)$ can be assumed proportional to the difference between the demand $D(p)$ and the supply $S(p)$ functions; that is,

$$
\dot{p}=\beta[D(p)-S(p)],
$$

where $\beta$ is proportionally constant. Let $p=p^{*}$ be the equilibrium price; then

$$
D\left(p^{*}\right)=S\left(p^{*}\right) .
$$

Next a fast oscillating periodic force with zero mean is applied to the dynamics of the market; then (10) takes the form

$$
\begin{aligned}
\dot{p}= & \beta[D(p)-S(p)] \\
& +\sum_{k=1}^{\infty}\left(a_{k} \cos k \omega t+b_{k} \sin k \omega t\right) .
\end{aligned}
$$

Here $\omega=2 \pi / T \gg \omega_{0}=2 \pi / T_{0}$ is the frequency of market perturbation with $\omega_{0}$ which is the approaching speed of (10) towards equilibrium price. In (12),

$$
\begin{aligned}
& a_{k}=a_{k}(p) \\
& b_{k}=b_{k}(p)
\end{aligned}
$$

are price dependent amplitudes.

Like Kapitza method, the price $p(t)$ can be split into its slow $\phi(t)$ and fast $\xi(t)$ components as

$$
p(t)=\phi(t)+\xi(t)
$$

and its time derivative is

$$
\dot{p}=\dot{\phi}+\dot{\xi}
$$

We assume that $a_{k}=a_{k}(\phi)$ and $b_{k}=b_{k}(\phi)$. Using above transformations defined in (14) and (15), (12) becomes

$$
\begin{aligned}
\dot{\phi}+\dot{\xi}= & \beta[D(\phi+\xi)-S(\phi+\xi)] \\
& +\sum_{k=1}^{\infty}\left(a_{k} \cos k \omega t+b_{k} \sin k \omega t\right) .
\end{aligned}
$$

Next using Taylor's series expansion up to 2nd order term, (16) can be rewritten as

$$
\begin{aligned}
\dot{\phi}+\dot{\xi}= & F(\phi)+\xi F^{\prime}(\phi)+\frac{1}{2} \xi^{2} F^{\prime \prime}(\phi) \\
& +\sum_{k=1}^{\infty}\left(a_{k} \cos k \omega t+b_{k} \sin k \omega t\right),
\end{aligned}
$$

where $F(\phi)=\beta[D(p)-S(p)]$. 


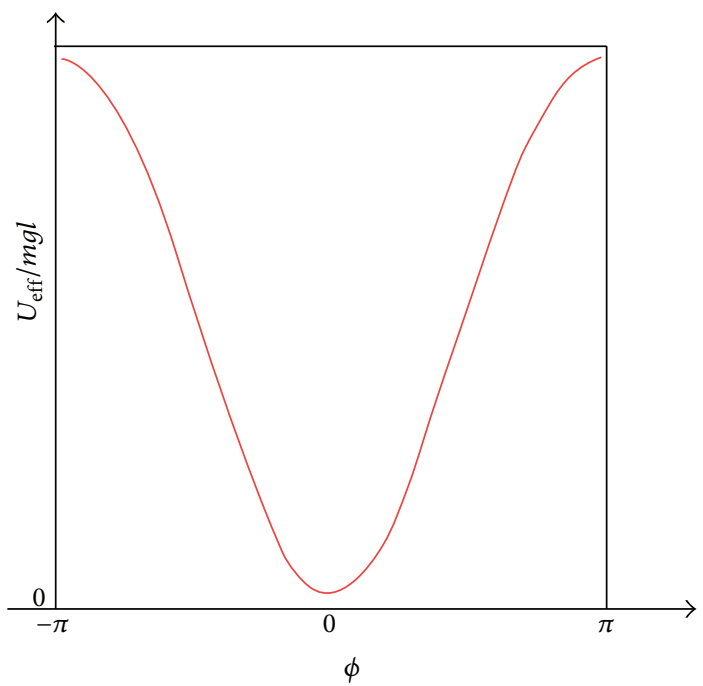

(a)

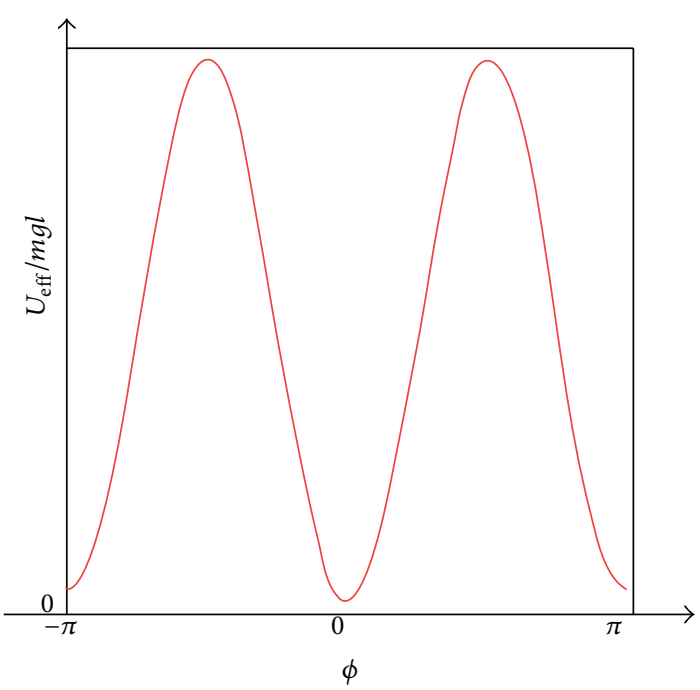

(b)

FIGURE 2: Minimization of dimensionless effective potential energy function.

Now the slow and fast part must be separately equal; for fast part we simply put

$$
\dot{\xi}=\sum_{k=1}^{\infty}\left(a_{k} \cos k \omega t+b_{k} \sin k \omega t\right) \text {. }
$$

Integrating (18) with initial condition $\xi_{0}=0$, the fast component is

$$
\xi=\frac{1}{\omega} \sum_{k=1}^{\infty} \frac{1}{k}\left(a_{k} \sin k \omega t-b_{k} \cos k \omega t\right)
$$

Using (3), the mean values of $\xi$ and $\dot{\xi}$ are zero, while $\phi$ and $\dot{\phi}$ are unaltered. Then the mean values of (14) and (15) can be given as

$$
\begin{aligned}
& \bar{p}=\phi \\
& \bar{p}=\dot{\phi} .
\end{aligned}
$$

Hence $\phi$ describes the slow price and $\dot{\phi}$ describes the rate of slow price averaged over rapid oscillations. are

Next the mean values of $\xi^{2}$ over the time interval $[0, T]$

$$
\overline{\xi^{2}}=\frac{1}{2 \omega^{2}} \sum_{k=1}^{\infty}\left(\frac{a_{k}^{2}+b_{k}^{2}}{k^{2}}\right)
$$

Also $F(\phi)$ and its derivatives remain unaltered during this time averaging. Next time averaging of (17) will give a function of $\phi$ only. That is,

$$
\dot{\phi}=F(\phi)+\frac{1}{4 \omega^{2}} \sum_{k=1}^{\infty}\left(\frac{a_{k}^{2}+b_{k}^{2}}{k^{2}}\right) F^{\prime \prime}(\phi)
$$

or

$$
\overline{\dot{p}}=\beta\left([D-S](\phi)+\frac{1}{4 \omega^{2}} \sum_{k=1}^{\infty}\left(\frac{a_{k}^{2}+b_{k}^{2}}{k^{2}}\right)\left[D^{\prime \prime}-S^{\prime \prime}\right](\phi)\right) .
$$

It shows that after averaging, the influence of fast periodic oscillations on the slow price component is also depending on curvatures of demand and supply functions, and this effect vanishes when both functions are linear. Due to this fast oscillation, the averaged equilibrium price can be easily calculated. Expanding first term on right hand side of (23) into the power series around the value $p^{*}$ and considering linear part only, we have

$$
\begin{aligned}
\bar{p}=\beta( & {\left[D^{\prime}(\phi)-S^{\prime}(\phi)\right]\left(\phi-\phi^{*}\right) } \\
& \left.+\frac{1}{4 \omega^{2}}\left[D^{\prime \prime}(\phi)-S^{\prime \prime}(\phi)\right] \sum_{k=1}^{\infty}\left(\frac{a_{k}^{2}+b_{k}^{2}}{k^{2}}\right)\right) .
\end{aligned}
$$

Following (11) at equilibrium price, we must have $\overline{\dot{p}}=0$,

$$
\begin{aligned}
0=\beta( & {\left[D^{\prime}(\phi)-S^{\prime}(\phi)\right]\left(\phi-\phi^{*}\right) } \\
& \left.+\frac{1}{4 \omega^{2}}\left[D^{\prime \prime}(\phi)-S^{\prime \prime}(\phi)\right] \sum_{k=1}^{\infty}\left(\frac{a_{k}^{2}+b_{k}^{2}}{k^{2}}\right)\right) ;
\end{aligned}
$$

consequently,

$$
\phi-\phi^{*}=\frac{1}{4 \omega^{2}} \frac{D^{\prime \prime}(\phi)-S^{\prime \prime}(\phi)}{S^{\prime}(\phi)-D^{\prime}(\phi)} \sum_{k=1}^{\infty}\left(\frac{a_{k}^{2}+b_{k}^{2}}{k^{2}}\right) .
$$

The right hand side of (26) is a function of $\phi$ only. Since after averaging $\phi$ changes slightly, that is, $\bar{\phi} \approx \phi$. Also at 
equilibrium, $\phi$ is very near to $\phi^{*}$, while $\overline{p^{*}}$ can be located far from $p^{*}$. Hence for good approximation, we can write

$$
\overline{p^{*}}-p^{*}=\Delta p^{*}=\frac{1}{4 \omega^{2}} \frac{D^{\prime \prime}(p)-S^{\prime \prime}(p)}{S^{\prime}(p)-D^{\prime}(p)} \sum_{k=1}^{\infty}\left(\frac{a_{k}^{2}+b_{k}^{2}}{k^{2}}\right) .
$$

Equation (27) gives the shift of equilibrium price of the market due to fast oscillations.

This shift can increase the equilibrium price of a typical market if

(a) the infinite sum increases;

(b) the frequency of small oscillation is small;

(c)

$$
\frac{D^{\prime \prime}(p)-S^{\prime \prime}(p)}{S^{\prime}(p)-D^{\prime}(p)}>0
$$

In Kapitza method of averaging for arbitrary periodic force, the increase in infinite sum will decrease the frequency of oscillation at $\phi=\pi$. To follow (28), we must suppose that $D^{\prime}(p)<0 ; D^{\prime \prime}(p)>0 ; S^{\prime}(p)>0$ and $S^{\prime \prime}(p)<0$. Then it follows that, as price $p(t)$ increases, the demand $D(p)$ decreases and $S(p)$ increases in a slow manner. As a result, saturation in demand and supply is established. Hence by averaging procedure the equilibrium price has shifted up. Choose

$$
\frac{D^{\prime \prime}(p)-S^{\prime \prime}(p)}{S^{\prime}(p)-D^{\prime}(p)}=C^{2}>0 .
$$

Then (27) can be rewritten as

$$
\begin{aligned}
\Delta p^{*} & =\frac{1}{4 \omega^{2}} C^{2} \sum_{k=1}^{\infty}\left(\frac{a_{k}^{2}+b_{k}^{2}}{k^{2}}\right) \\
& =0.25 A S_{k},
\end{aligned}
$$

where $A=C^{2} / \omega^{2}$.

\section{Shift of Market Equilibrium by External Arbitrary Periodic Force}

In this section we will select some arbitrary periodic forces and study the increase in equilibrium price by using (30).

4.1. Harmonic Force. First we choose the harmonic force

$$
f(t)=\sin \omega t
$$

as external force (see Figure 3). Using (4), the Fourier coefficient $a_{0}=0$ indicates that the mean value of $f(t)$ about its period $T=2 \pi / \omega$ is zero. Next using (6), the other Fourier coefficients for (31) are

$$
\begin{gathered}
a_{k}=0 \\
b_{k}= \begin{cases}0 & k \neq 1 \\
1 & k=1 .\end{cases}
\end{gathered}
$$

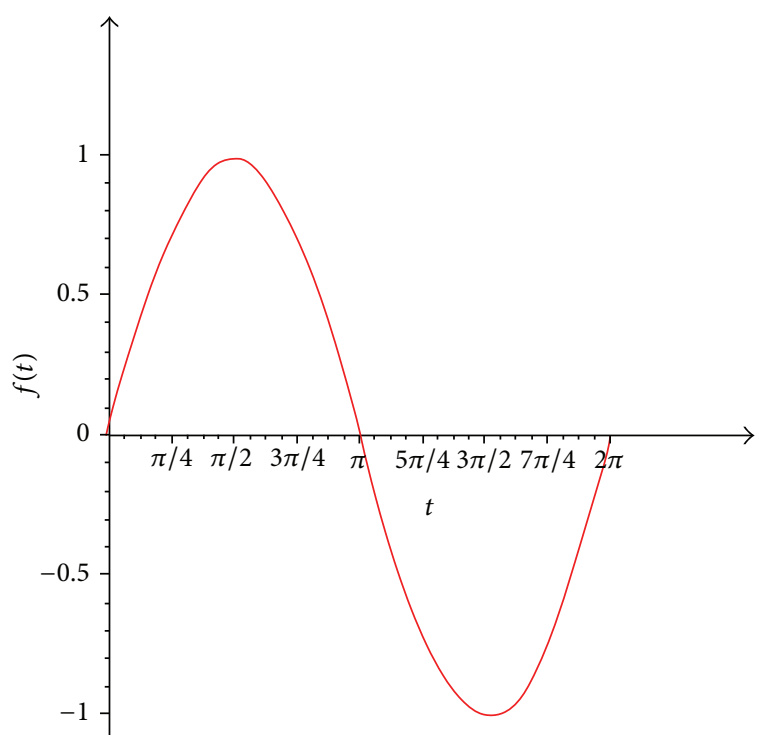

FIGURE 3: Sine type external force.



Equilibrium price due to external periodic force

Equilibrium price without external periodic force

FIGURE 4: Change in equilibrium due to external force.

Using these coefficients in (30), the old equilibrium price is shifted to new equilibrium price by [14]

$$
\Delta p^{*}=0.25 A \text {. }
$$

This shift is illustrated in Figure 4.

4.2. Triangular Force. Next we apply periodical triangular type force $R_{s}(t)=R_{s}(t+T)$

$$
R_{S}(t)= \begin{cases}\frac{4 t}{T} & \text { if } 0 \leq t<\frac{T}{4} ; \\ \frac{4}{T}\left(\frac{T}{2}-t\right) & \text { if } \frac{T}{4} \leq t<\frac{3 T}{4} \\ \frac{4(t-T)}{T} & \text { if } \frac{3 T}{4} \leq t<T,\end{cases}
$$


with the same property $\bar{R}_{S}=0$ (see Figure 5). Next using (6), the other Fourier coefficients for (34) are

$$
\begin{gathered}
a_{k}=0 \\
b_{k}=\frac{4}{k^{2} \pi^{2}}\left[1-(-1)^{k}\right]
\end{gathered}
$$

or

$$
b_{k}= \begin{cases}0 & k \text { is even } \\ \frac{8}{k^{2} \pi^{2}} & k \text { is odd }\end{cases}
$$

Using these coefficients in (30), the shift in equilibrium price is

$$
\begin{aligned}
\Delta p^{*} & =0.25 A \frac{64}{\pi^{4}} \sum_{k=1}^{\infty} \frac{1}{(2 k-1)^{6}} \\
& =\frac{16}{\pi^{4}} \frac{\pi^{6}}{960} A \\
& =0.164 A .
\end{aligned}
$$

Here (37) gives the shift of new equilibrium price from the old one due to fast oscillation with triangular external force. This increase is lower than the increase with harmonic force.

4.3. Rectangular Force. The next force is rectangular type force $R_{l}(t)=R_{l}(t+T)$ (see Figure 6), given by

$$
R_{l}(t)= \begin{cases}1 & 0 \leq t \leq \frac{T}{2} \\ -1 & \frac{T}{2} \leq t \leq T\end{cases}
$$

with the same property $\bar{R}_{l}=0$.

Next using (6), the other Fourier coefficients for (38) are

$$
\begin{gathered}
a_{k}=0 \\
b_{k}= \begin{cases}0 & k \text { is even } \\
\frac{4}{k \pi} & k \text { is odd }\end{cases}
\end{gathered}
$$

or

$$
b_{2 k-1}=\frac{4}{(2 k-1) \pi} .
$$

Using these coefficients in (30), the shift in equilibrium price is

$$
\begin{aligned}
\Delta p^{*} & =0.25 A \frac{16}{\pi^{2}} \sum_{k=1}^{\infty} \frac{1}{(2 k-1)^{4}} \\
& =0.25 A(1.645) \\
& =0.411 A .
\end{aligned}
$$

Here (41) gives the shift of new equilibrium price for rectangular force. It has raised up the equilibrium price than the previous forces. Hence by applying a different force, an increase/decrease in equilibrium price is possible.

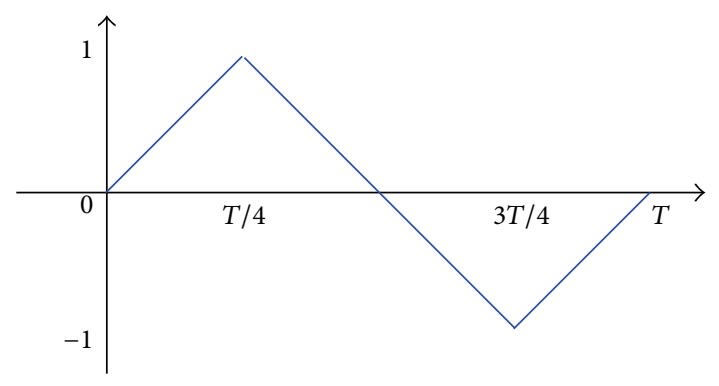

Figure 5: Triangular type force.

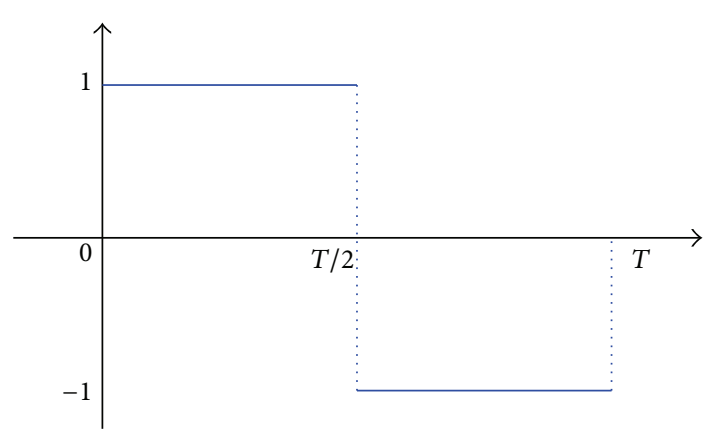

FIGURE 6: Rectangular type force.

\section{Conclusions}

A method similar to Kapitza method of averaging for an arbitrary periodic force is used in the dynamics of the market. Then another equilibrium price with an increase may be possible. Previously harmonic force was used to raise the equilibrium price of the market. In this work an arbitrary periodic force with zero mean is applied to raise it. Now this increase can be controlled by varying the external arbitrary periodic force. On the same pattern, when the government needs money, it announces an increase in the rates of existing taxes or adds new taxes, and when it wants to give relief to public, it decreases the rates of existing taxes or deletes some taxes in the price of commodity.

In September 2012, compressed natural gas (CNG) was being sold at Rs. 92.53 per $\mathrm{kg}$ in region I. Details are in Table 1 [16].

In Table 1 , if $A$ was gas price, then some external factors were involved and $E$ was the next price; again price was decided by adding some factors (federal price, compression cost, profit, and taxes) and the consumer price was $L$. Here $E$ and $L$ were new equilibrium prices with an increase.

Next Consumer Rights Commission of Pakistan did not consider it a justified price, so they pursued the Supreme Court of Pakistan, who on October 26, 2012 passed an order, declaring to implement the July 1, 2012 prices. That price detail is in Table 2 [17].

Then the consumer was paying Rs. 61 instead of Rs. 92.53 per $\mathrm{kg}$ in region $\mathrm{I}$. 
TABLE 1: CNG consumer price detailed break up w.e.f. October 22, 2012.

\begin{tabular}{|c|c|c|c|c|c|c|c|}
\hline \multirow{2}{*}{ S number } & \multirow{2}{*}{ Components } & \multicolumn{3}{|c|}{ Region I } & \multicolumn{3}{|c|}{ Region II } \\
\hline & & Rs./Kg & Rs.MMBtu & $\%$ & Rs./Kg & Rs.MMBtu & $\%$ \\
\hline$A$ & $\begin{array}{l}\text { Average well head } \\
\text { price/cost of gas paid to } \\
\text { exploration companies }\end{array}$ & 18.04 & 358.87 & $92 \%$ & 16.48 & 358.87 & $92 \%$ \\
\hline$B$ & $\begin{array}{l}\text { Operating cost of gas } \\
\text { companies }\end{array}$ & 1.14 & 22.69 & $6 \%$ & 1.04 & 22.69 & $6 \%$ \\
\hline C & $\begin{array}{l}\text { Return on investment to } \\
\text { gas companies Other } \\
\text { incomes (net of prior year }\end{array}$ & 18.04 & 358.87 & $92 \%$ & 16.48 & 358.87 & $92 \%$ \\
\hline$D$ & adjustment, if any, etc.) & -0.53 & -10.63 & $-3 \%$ & -0.49 & -10.63 & $-3 \%$ \\
\hline$E=A$ to $D$ & $\begin{array}{l}\text { Average prescribed price of } \\
\text { natural gas }\end{array}$ & 19.51 & 388.27 & $100 \%$ & 17.83 & 388.26 & $100 \%$ \\
\hline$F$ & $\begin{array}{l}\text { Sale price of natural gas for } \\
\text { CNG stations as advised by } \\
\text { Federal Government under } \\
\text { Section } 8(3) \text { of OGRA, } \\
\text { ordinance including } \\
\text { GDS/cross-subsidization. }\end{array}$ & 35.19 & 700.32 & $38 \%$ & 32.14 & 700.02 & $38 \%$ \\
\hline G & $\begin{array}{l}\text { Operating cost of gas } \\
\text { stations }\end{array}$ & 20.80 & 113.80 & $22 \%$ & 20.80 & 453.01 & $25 \%$ \\
\hline$H$ & $\begin{array}{l}\text { Profit of CNG station } \\
\text { owners. }\end{array}$ & 11.19 & 222.60 & $12 \%$ & 10.59 & 230.61 & $13 \%$ \\
\hline$I$ & GIDC & 13.25 & 263.57 & $14 \%$ & 9.18 & 200.00 & $11 \%$ \\
\hline$J$ & GST $(25 \%$ of price $F+1)$ & 12.10 & 240.69 & $13 \%$ & 10.33 & 225.00 & $12 \%$ \\
\hline K & $\begin{array}{l}\text { Differential margin for } \\
\text { region II }\end{array}$ & - & & & 1.50 & 32.67 & $2 \%$ \\
\hline$L$ & $\begin{array}{l}\text { Total CNG consumer price } \\
(F-K)\end{array}$ & 92.53 & $1,840.98$ & $100 \%$ & 84.54 & $1,841.31$ & $100 \%$ \\
\hline
\end{tabular}

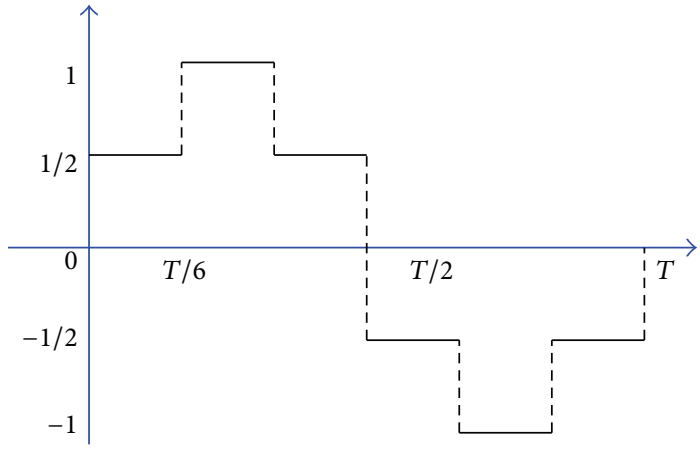

Figure 7: Hat type force.

In Table 1, the equilibrium price was raised up by adding some factors, and in Table 2 it was lowered down by deleting some factors. So when the authority needs money it raised up the prices, and when it wants to give relieve to people, it lowers down the prices.

A number of more examples can be found. Every consumer has to pay Rs. 35 as ptv. (Pakistan television) fee, and to support Neelum Jhelum project, they are bearing the cost of delay and inefficiency and are paying a surcharge of 10 paisa per unit in their electricity bills. Moreover different slabs of usage units have different rates of billing.

\section{Appendices}

Here some more periodic forces with zero mean are given.

\section{A. Hat Force}

The first force is rectangular hat type (see Figure 7), defined by

$$
L_{c}(t)=\left\{\begin{aligned}
\frac{1}{2} & \text { if } 0 \leq t<\frac{1}{6} T \\
1 & \text { if } \frac{1}{6} T \leq t<\frac{1}{3} T \\
\frac{1}{2} & \text { if } \frac{1}{3} T \leq t<\frac{1}{2} T \\
-\frac{1}{2} & \text { if } \frac{1}{2} T \leq t<\frac{2}{3} T \\
-1 & \text { if } \frac{2}{3} T \leq t<\frac{5}{6} T \\
-\frac{1}{2} & \text { if } \frac{5}{6} T \leq t<T
\end{aligned}\right.
$$


TABLE 2: CNG consumer price detailed break up.

S number Components of price

Region I Region II

Cost of production

(a) Cost of gas billed

$31.09 \quad 28.40$

(b) Cost of compression

$5.46 \quad 5.46$

total cost of production

$36.55 \quad 33.86$

2

(10-12\%) Retailer fixed profit

4.386

4.063

Taxes

3

(a) GIDC $13 \quad 13$

(b) GST $21 \%$ cost of gas

6.529

5.964

$19.529 \quad 18.964$

Total $(1+2+3)$

$60.465 \quad 56.887$

5 Consumer retail price (consumer willingness to pay)

61

57

Its mean value about its period is zero. Then by Fourier expansion in place of (A.1),

$$
\begin{gathered}
a_{k}=0 \\
b_{k}=\frac{1}{k \pi}\left(1-\cos k \pi+2 \cos k \frac{\pi}{3}\right) .
\end{gathered}
$$

Using these coefficients in (30), the shift in equilibrium price is

$$
\begin{aligned}
\Delta p^{*} & =0.25 A \sum_{k=1}^{\infty} \frac{1}{k^{2}}\left[\frac{1}{k \pi}\left(1-\cos k \pi+2 \cos k \frac{\pi}{3}\right)\right]^{2} \\
& =0.25 A(0.9208) \\
& =0.2302 A .
\end{aligned}
$$

This force also lowered down the equilibrium price as compared to harmonic force but raised up the triangular type force.

\section{B. Trapezoidal Force}

The next force is trapezoidal type force $T_{m}(t)=T_{m}(t+T)$ (see Figure 8), given by

$$
T_{m}(t)= \begin{cases}\frac{8 t}{T} & \text { if } 0 \leq t<\frac{T}{8} ; \\ 1 & \text { if } \frac{T}{8} \leq t<\frac{3 T}{8} ; \\ \frac{8}{T}\left(\frac{T}{2}-t\right) & \text { if } \frac{3 T}{8} \leq t<\frac{5 T}{8} ; \\ -1 & \text { if } \frac{5 T}{8} \leq t<\frac{7 T}{8} ; \\ \frac{8(t-T)}{T} & \text { if } \frac{7 T}{8} \leq t<T,\end{cases}
$$

since $a_{0}=0 \Rightarrow \bar{T}_{m}=0$.

Next the Fourier coefficients of (B.1) are

$$
\begin{gathered}
a_{k}=0 \\
b_{k}=\frac{16}{\pi^{2}} \frac{1}{k^{2}} \sin k \frac{\pi}{4} .
\end{gathered}
$$

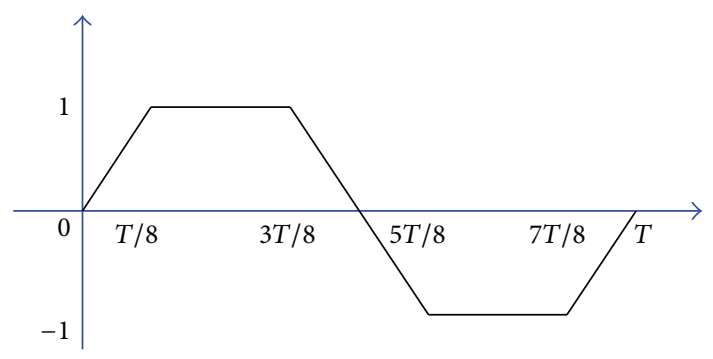

Figure 8: Trapezoidal type force.

Using these coefficients in (30), the shift in equilibrium price is

$$
\begin{aligned}
\Delta p^{*} & =0.25 A \frac{256}{\pi^{4}} \sum_{k=1}^{\infty} \frac{1}{k^{6}} \sin ^{2} k \frac{\pi}{4} \\
& =0.25 A(1.3571) \\
& =0.3393 A .
\end{aligned}
$$

Due to this force the equilibrium price has been raised up as compared to hat type force.

\section{Quadratic Force}

The next force is quadratic type force: $Q_{c}(t)=Q_{c}(t+T)$ (see Figure 9), given by

$$
Q_{c}(t)= \begin{cases}1 & \text { if } 0 \leq t<\frac{3 T}{8} \\ \frac{8}{T}\left(\frac{T}{2}-t\right) & \text { if } \frac{3 T}{8} \leq t<\frac{5 T}{8} \\ -1 & \text { if } \frac{5 T}{8} \leq t<T\end{cases}
$$

with the same property $\bar{Q}_{c}=0$ as $a_{0}=0$. Then by Fourier expansion in the place of (C.1)

$$
\begin{gathered}
a_{k}=0 \\
b_{k}=\left(\frac{2}{k \pi}+\frac{8}{\pi^{2} k^{2}} \sin k \frac{\pi}{4}\right) .
\end{gathered}
$$

Using these coefficients in (30), the shift in equilibrium price is

$$
\begin{aligned}
\Delta p^{*} & =0.25 A \sum_{k=1}^{\infty} \frac{1}{k^{2}}\left(\frac{2}{k \pi}+\frac{8}{\pi^{2} k^{2}} \sin k \frac{\pi}{4}\right)^{2} \\
& =0.25 A(1.5426) \\
& =0.3857 A .
\end{aligned}
$$

It is observed that applying this external force, the equilibrium price has more raised up.

All these results with conditional stable points are given in Table 3.

By applying a different force, an increase/decrease in equilibrium price can be made. 


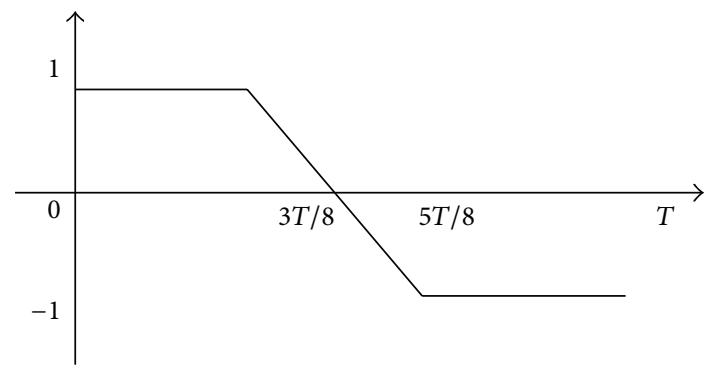

FIgURE 9: Quadratic type force.

TABLE 3: Shift in price equilibrium by fast oscillation.

\begin{tabular}{lccc}
\hline Force type & $\begin{array}{c}\text { Sum } \\
\sum_{k=1}^{\infty} \frac{\left(a_{k}^{2}+b_{k}^{2}\right)}{k^{2}}\end{array}$ & $\begin{array}{c}\text { Stability } \\
\text { condition at } \phi=\pi\end{array}$ & $\begin{array}{c}\text { Shift in price } \\
\text { Equilibrium } \Delta p^{*}\end{array}$ \\
\hline Sin & 1 & $\omega^{2}>2 g l$ & $0.25 \mathrm{~A}$ \\
Triangular & 0.658 & $\omega^{2}>3.0396 g l$ & $0.164 \mathrm{~A}$ \\
Linear hat & 0.9208 & $\omega^{2}>2.172 g l$ & $0.2302 \mathrm{~A}$ \\
Trapezium & 1.3571 & $\omega^{2}>1.4736 g l$ & $0.3393 \mathrm{~A}$ \\
Quadratic & 1.5426 & $\omega^{2}>1.2967 g l$ & $0.3857 \mathrm{~A}$ \\
Rectangular & 1.645 & $\omega^{2}>1.2159 \mathrm{gl}$ & $0.411 \mathrm{~A}$ \\
\hline
\end{tabular}

\section{Conflict of Interests}

The authors declare that there is no conflict of interests regarding the publication of this paper.

\section{References}

[1] R. N. Mantegna and H. E. Stanley, An Introduction to Econophysics: Correlations and Complexity in Finance, Cambridge University Press, Cambridge, UK, 1999.

[2] R. N. Mantegna and H. E. Stanley, An Introduction to Econophysics: Correlations and Complexity in Finance, Cambridge University Press, Cambridge, UK, 2000.

[3] J.-P. Bouchaud, "An introduction to statistical finance," Physica A, vol. 313, no. 1-2, pp. 238-251, 2002.

[4] M. G. Daniels, J. D. Farmer, L. Gillemot, G. Iori, and E. Smith, "Quantitative model of price diffusion and market friction based on trading as a mechanistic random process," Physical Review Letters, vol. 90, Article ID 108102, 2003.

[5] R. Friedmann and W. G. Sanddorf-Köhle, "Volatility clustering and nontrading days in Chinese stock markets," Journal of Economics and Business, vol. 54, no. 2, pp. 193-217, 2002.

[6] G. Bonanno, D. Valenti, and B. Spagnolo, "Mean escape time in a system with stochastic volatility," Physical Review E, vol. 75, Article ID 016106, 2007.

[7] D. Eliezer and I. I. Kogan, "Scaling laws for the market microstructure of the interdealer broker markets," SSRN eLibrary, 1998.

[8] X. Yiping, R. Chandramouli, and C. Cordeiro, "Price dynamics in competitive agile spectrum access markets," IEEE Journal on Selected Areas in Communications, vol. 25, no. 3, pp. 613-621, 2007.
[9] D. Valenti, B. Spagnolo, and G. Bonanno, "Hitting time distributions in financial markets," Physica A, vol. 382, no. 1, pp. 311-320, 2007.

[10] H. Mizuta, K. Steiglitz, and E. Lirov, "Effects of price signal choices on market stability," Journal of Economic Behavior and Organization, vol. 52, no. 2, pp. 235-251, 2003.

[11] G. Bonanno, D. Valenti, and B. Spagnolo, "Role of noise in a market model with stochastic volatility," European Physical Journal B, vol. 53, no. 3, pp. 405-409, 2006.

[12] P. L. Kapitza, "Dynamic stability of a pendulum with an oscillating point of suspension," Journal of Experimental and Theoretical Physics, vol. 21, pp. 588-597, 1951.

[13] L. D. Landau and E. M. Lifshitz, Mecanics, Pergamon Press/Butterworth, Oxford, UK, 3rd edition, 2005.

[14] J. A. Hołyst and W. Wojciechowski, "The effect of Kapitza pendulum and price equilibrium," Physica A, vol. 324, no. 1-2, pp. 388-395, 2003.

[15] B. Ahmad and S. Borisenok, "Control of effective potential minima for Kapitza oscillator by periodical kicking pulses," Physics Letters A, vol. 373, no. 7, pp. 701-707, 2009.

[16] http://supremecourt.gov.pk/web/user_files/File/CONST.P.3334-2005.pdf.

[17] "CRCP House Islamabad," Islamabad, Pakistan, http://www .crcp.org.pk. 

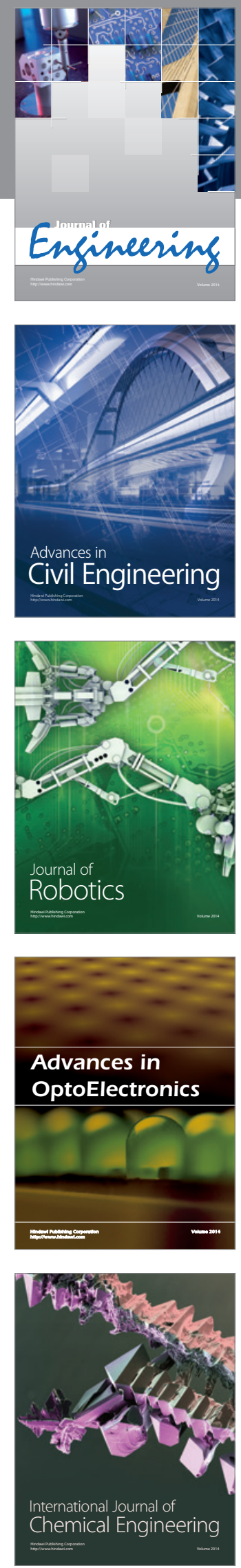

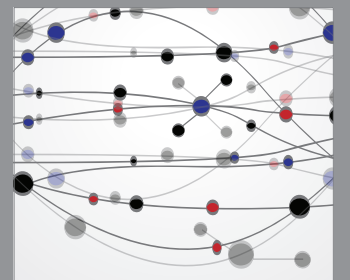

The Scientific World Journal
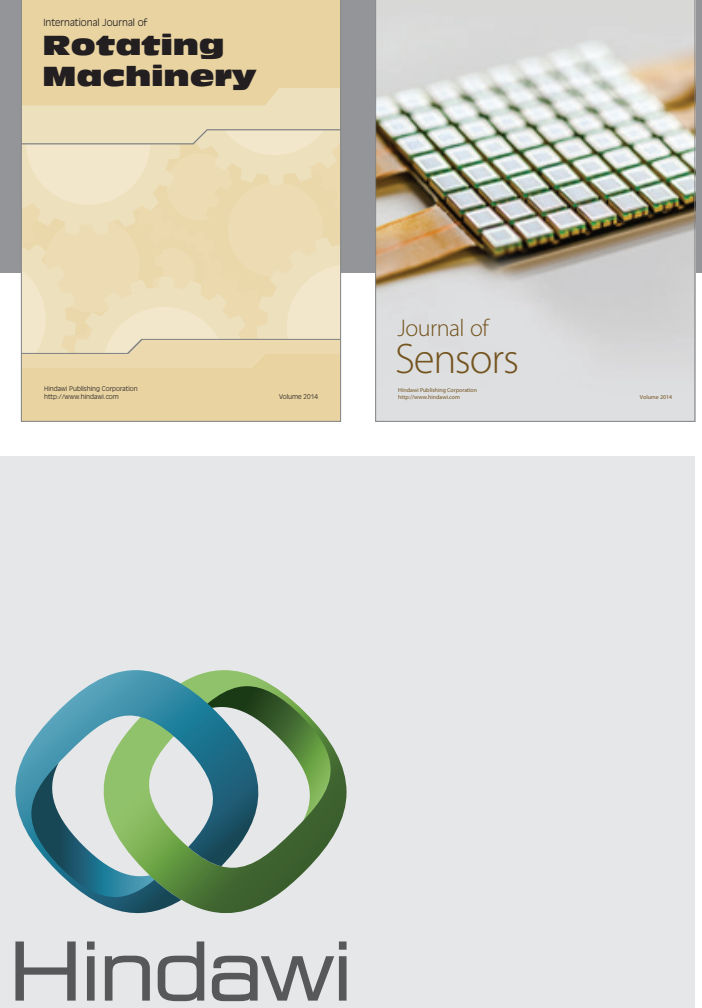

Submit your manuscripts at http://www.hindawi.com
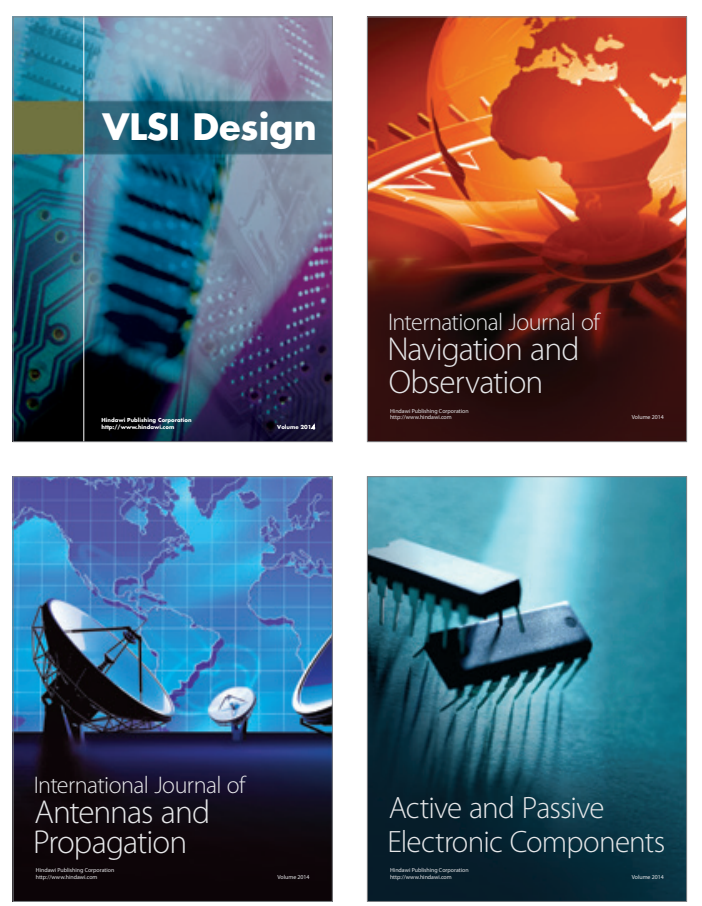
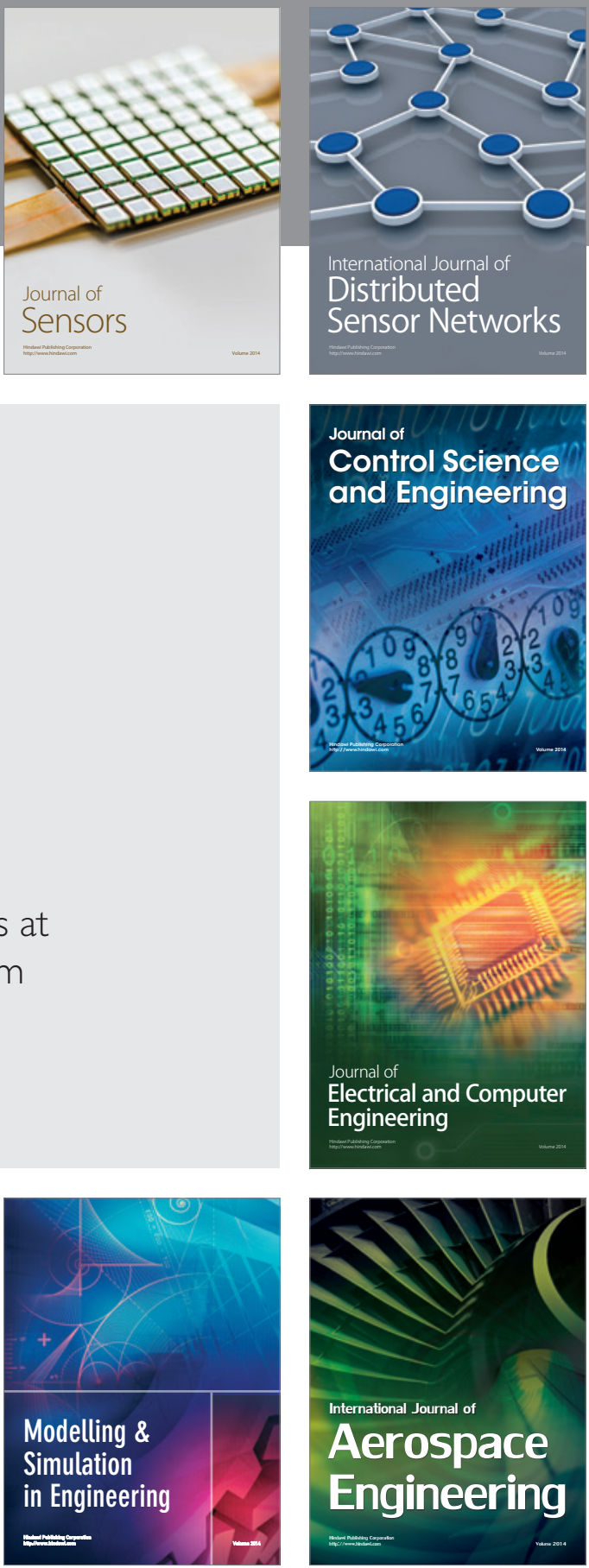

Journal of

Control Science

and Engineering
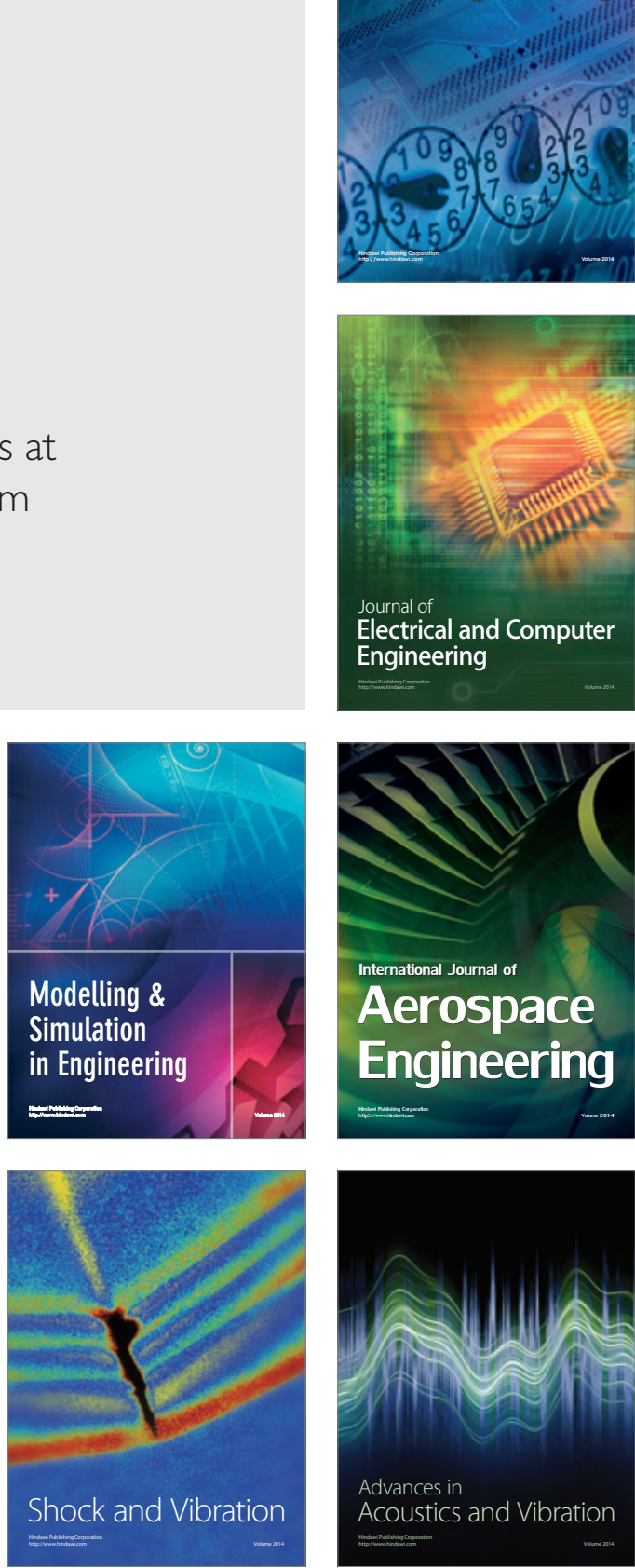